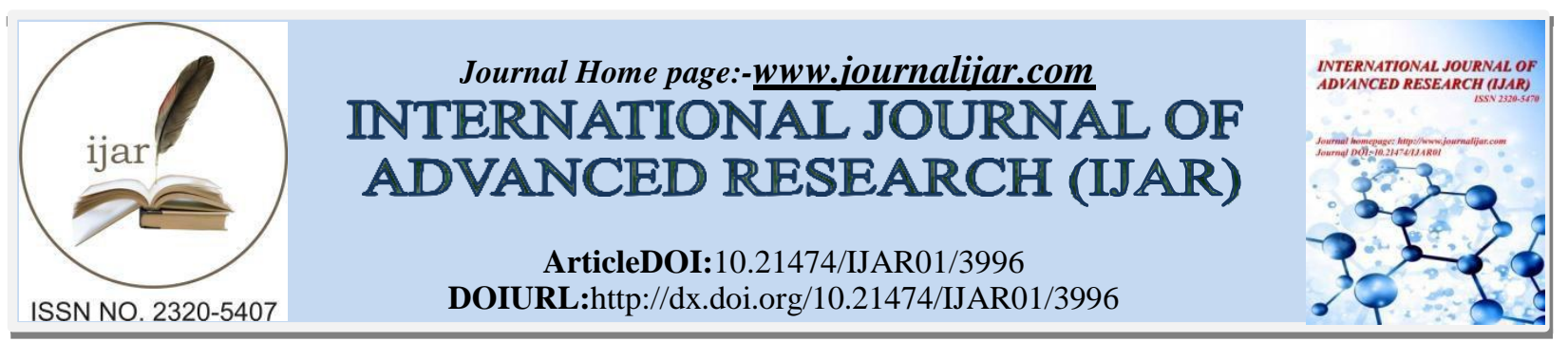

RESEARCH ARTICLE

\title{
MULTIPATH ROUTING AS A TOOL TO IMPROVE QUALITY OF SERVICES IN MANETS.
}

\author{
Dvss. Subrahmanyam ${ }^{1}$ and Dr. T. Ch. MalleswaraRao ${ }^{2}$ \\ 1. Research Scholar, Rayalaseema University, Kurnool. \\ 2. Scientist (Retd.), Nrsc, Isro, Dept .Of Space, Govt. Of India.
}

\section{Manuscript Info}

Manuscript History

Received: 05 February 2017

Final Accepted: 10 March 2017

Published: April 2017

Key words:-

Multipath routing, Ad hoc networks, MANETs , Quality of Services

\begin{abstract}
Wireless networks have become more prominent networks now-a-days because of their dynamic characteristics and flexibility. Ad hoc networks can be created anywhere / any time as per the needs and requirements of the existing situations. Mobile Ad hoc networks (MANETs) are invented to meet the goals and challenges of mobile devices. Because of their troubleshooting mechanism, emergency services, cost effectiveness of their infrastructure, consuming minimum amount of time for network establishment, penetrating capacity to enter into remote areas where normal communication lines do not exist etc., MANETs have become the centre point of the present communication networks systems. Thus much attention is needed to improve the quality of MANETs to get high efficient and effective services. Multipath routing is a good technical tool to improve the quality of MANETs in all aspects.
\end{abstract}

Copy Right, IJAR, 2017,.All rights reserved.

\section{Introduction:-}

Wireless networks were recommended by IEEE standard 802.11. But it is restricted to single-hop communications. As per the basic definitions are concerned, single-hop communication domains have many constraints. Single communication path exists between sender and receiver to send or receive packets. This concept works good as long as there is no heavy traffic between nodes and the communication path is not failed / broken. If any one condition is not met with then the entire network system will be broken down which leads to more waiting period to send data and the network communication might be collapsed. To overcome all these limitations of single-hop communication system, multi-hop or multiple-hop communication system is introduced which is the basic platform for Ad hoc networks (S.MuellerandD.Ghosal,2004). This system contains, many mobile nodes where the communication range of every mobile node differs from each and every other node of the system with respect to their node characteristics on the basis of their power consumption, bandwidth etc., In this network topology concept, direct communication is not possible between any two mobile nodes unless they are consecutive or neighbouring nodes.

Intermediate nodes play an important role to mediate between neighbouring nodes and they behave as network routers to fecilitate smooth functioning of communication process. The basic need of any communication system is that the communication path is to be established between mobile nodes (even in very high risk emergency services and battle field operations) (J.X.Wangetal,2006). MANETs always form an arbitrary network topology. It is highly dynamic in nature. Every time new mobile nodes may be added or existing nodes may be terminated (DimitriosLiarokapisetal,2004; ImrichChlamtacetal,2003). Thus it is a self-configured network topology which changes rapidly and unpredictably from time to time. New mobile nodes may join, unexpectedly, the existing 
network (C. Siva Rama Murty and B.S.Manoj,2004). The entire communication system is based on its communication paths. Designing network protocols for MANETs is very difficult because of their dynamic nature (A.B. McDonald and T.Zanti,1999) when compared with wired networks.

MANETs use single path routing communication for data transmissions between source and destination nodes. As long as the data that the mobile nodes sending and receiving are limited and participating mobile nodes are very few, no hurdles occur. But if the participating mobile nodes are big in number then the actual problems enter into these networks. All mobile nodes are supposed to share, only, the single existing communication path, so it may lead to congestion problems. More collisions of data packets may occur among the existing mobile nodes during their communication processes and so many other mobile nodes have to wait forcibly for their turn to transmit their data which may lead to unlimited waiting period for them. Ultimately the entire communication network system will be collapsed until it is renovated. So many measures are needed to bring the previous system into operation again. All these problems arise due to the utilization of single path routing communication systems. To overcome these disadvantages of single path routing, multipath routing is introduced.

Multipath routing is developed on the basis of multiple hops which is a combination of many single hop networks. In multipath routing many communication paths are to be established between any two mobile nodes because of the dynamic nature of mobile nodes. But at any time only one path will be in existence i.e., remaining paths will not work. If any path fails accidentally then any one of the remaining existing paths will be in force. The decision of the path to be used will be taken by the sending mobile node on the basis of the existing routing algorithms. At any time, only one communication path will be in force without throwing any problems to the network. Thus the probability of the existing communication network is always one. This is the strongest and fundamental concept of multipath routing which is highly recommended for ensuring quality of services in MANETs. Quality of services are, mainly, depending upon various parameters are discussed in detail as follows: i) Routing protocols ii )Security iii) Resources of the network iv) Reliable services of the network v) Energy saving vi) Location information management vii) Clustering viii) Automatic network configuration etc.,.

Routing protocols: These play an important role in deciding a new communication path between any two mobile nodes when the communication link fails between nodes. This is a very crucial step in which a spontaneous decision will be taken by the source node soon after it gets link failure message. Ad hoc On-demand Dynamic vector routing Protocol (AODV), Dynamic Source Routing Protocol (DSR), Zone Routing Protocol (ZRP) etc., are popularly used routing protocols. Temporally Ordered Routing Algorithm (TORA) is also widely used algorithm for routing protocols. Many routing protocols are existing in addition to them to take a decision about the communication path to be established but routing protocols to be chosen change from time to time depending upon the prevailing situation. Routing protocols exhibit high dynamics especially in situations such as battle fields, army attacks on enemies etc., (J. Hakoda et al, 2002). Routing protocols which are designed for wired networks are not suitable for wireless networks as they have control over head upon them. So wireless networks need specific and strong, in nature, routing protocols because of their high dynamic fluctuations in nature. But designing of routing protocols is not that much easier and highly complicated and their infrastructure requirements are different from that of wired networks (A.B. McDonald and T. Zanti, 1999).

Security issues: Many security measures are needed in case of wireless networks as they are prone to many network attacks. In some situations mobile nodes may not forward data packets to their next mobile node in communication process. Here their behavior can be suspected. As communication paths are wireless there is no guarantee of data packets to reach their exact destination. In many cases their probability of reaching the destination node is zero. This is the main door for attackers to enter into wireless network without the prior knowledge of the network. Attackers can divert the data packets from one node to another node which may belong to other network. In some cases attackers may inject their own data packets into the network. In all these cases the network may not have knowledge about their presence. These problems are existing especially in multipath rooting protocols when they establish new communication paths in case of failures. These special issues are to be addressed to provide a high quality of services in MANETs.

Resources of the network: Many mobile nodes in the network provide various services to the network including internet access. But all nodes do not know about these services provided to the network. Lack of knowledge of the network resources, mobile nodes may not be in a position to utilize them properly. This leads to non-functionality of 
properties properly. So a clear cut mechanism is needed to make all mobile nodes known about the resources provided by the network.

Reliable services of the network: These include fast establishment of network links, safety measures undertaken, ensuring safe transportation of data packets from source node to the destination node, the capability and power of the source node to receive communication link failure messages if any link fails during its communication process.

Energy saving: Distribution of data packets of mobile nodes is based on there sources available, power of mobile nodes, bandwidth etc., (Barritt Brain J et al, 2011). These parameters are not same to all mobile nodes but differs from node to node. Especially dissipation of data packets is based on the power factor available to mobile nodes. In general batteries happen to be the main power sources to mobile nodes. To perform operations for a long time the available power is to be utilized more effectively and efficiently. Any energy saving measures are to be adopted.

Location information management: In broad networks it is very difficult to find the location of link failure or the location of emergency services needed. Otherwise most of the time will be wasted for searching the actual location. Once it is known then most of the time could be saved by mobile nodes and they will be in a position to provide services efficiently and effectively.

Clustering: It is a type of nomenclature to divide all network mobile nodes into a finite number of groups called clusters. This ideology is helpful to networks in cases of providing emergency services, establishing connections when network links are failed, applying node mechanisms etc.,

Automatic network configuration: It is one of the basic features of wireless network topology. Mobile nodes live for short period and every time new nodes may be added and some existing nodes may be deleted because of their dynamic nature. Multipath routing has been the backbone structure of automatic network configuration. Efficiency and effectiveness of network's capabilities are based on this factor. It is also known as dynamic network configuration. At any instance of time communication path link exists between any two mobile nodes of the network and all these communication paths are from the source node to the destination node through many number of intermediate nodes. Power factor plays a vital role in automatic network configuration as the dissipation capability of the network is proportional to the available battery power in the network to nodes.

In MANETs source nodes use simple broadcasting technique called Flooding to distribute packets over any network. It attracts many problems in broadcasting but here it is not the main issue of this paper. But for the quality of service it is to be considered in its side-effects point of view. Because of this Flooding technique of data packets more collisions are possible during its transmission which could lead to degradation of the quality of the network performance in all aspects. So broadcasting is also to be considered for quality of services. Multipath routing not only improves efficiency of MANETs but also improves the performance of sensor networks too. Reliability of services is one of the main issues in MANETs. Mobile nodes in general select other nodes of good power to get transmitted their data packets. For smooth functioning of data transmission good energy sources also needed. Source nodes get known about the link failures by so many routing messages.

Latency factor also plays a vital role in deciding the quality factor of services provide by MANETs. The time taken by a data packet to travel from one node to another node is called Latency Factor. Quality of transmission degrades if latency factor is high. So for good quality of services the network has to ensure that it has very low latency factor for its transmissions. So many other types of delays are to be addressed such as propagation delay, transmission delay etc., among all networks. To get a good quality of service all these factors are to be minimized to their lowest levels. Latency protocols take and decide route destinations of the network. The rate of change in the amount of time a data packet takes is called Jitter. If jitter factor is high then latency factor will be minimized to send data packets to the destination node. Multipath routing reduces latency factor by increasing its jitter factor for all of its routing protocols to their minimum. That is why multipath routing techniques have been most widely popularized.

Multipath routing reduces serial occurrence of path errors during transmissions. This leads to low latency factors for improving the quality of services of MANETs. Since distances between mobile nodes is very long It is very critical and typical issue for the source node to ensure continuous transmission service to its users. So Global Positioning System (GPS) is introduced to look after all these problems arise during their transmissions. If any data packet fails to reach its destination the source node is supposed to transmit the data again. Many resources and 
power are wasted due to this reason. So ensuring safe and secured transmission is the primary responsibility of the network. Multipath routing also faces main problems due to their constant mobility of nodes and limited availability of bandwidth. When nodes are moving constantly, link availability problem is to be resolved in advance. If limited bandwidth is available then the network topology is to be modified. These two problems are to be addressed to resolve quality issues in MANETs.

Taking all factors into consideration, it is very clear that designing of multipath routing is a very difficult task in networks but it is the primary factor of deciding the quality of services in MANETs. Two types of communication links are existing i) stable links and ii) unstable links. So establishing a communication link is not an issue but establishing more stable links is very important for source nodes. If accidentally unstable links are formed then the entire network will be collapsed due to more communication link failures. So quality of communication link formation I s also a major issue in deciding the quality factor in MANETs. By considering all these open issues into account (there are many hidden parameters here it is not discussed in this paper) it can be stated that multipath routing can be used as a technical tool in providing and improving the quality of services in MANETs. In depth and detailed discussions are further needed to establish extensions of all these facts.

\section{References:-}

1. B.L.Sun,C.Gui,Q.F.Zhang,H.Chen,"FuzzyControllerBasedQoSRoutingAlgorithmwithaMulticlassSchemeforMANET, "InternationalJournalofComputers,Communications\&Control,Vol.IV,No.4,2009,pp.427-438.

2. BannerR.,OrdaA..Multi-path Routing Algorithms for Congestion Minimization .IEEE/ACM Transactions onNetworking,vol.15(2),April2007, pages413-424.

3. Barritt, BrianJ.; Sheikh, Shaya; Al-Najjar, Camelia; Malakooti, Behnam "Mobile ad hoc network broadcasting: Amulti-criteria approach", International Journal of Communication Systems, Vol.24,Issue4,pp.438-460,April2011.

4. B.L.Sun,C.Gui,Q.F.Zhang, et al ,"A Multipath on-Demand Routing with Path Selection Entropy for Ad Hoc Networks, " Proceedings of The $9^{\text {th }}$ International Conference for Young Computer Scientists (ICYCS2008), ZhangJiajie,Hunan,China,18-21November,2008,pp.558-563.

5. $\mathrm{P} \quad \mathrm{Ng}, \mathrm{S}$ Liew. Throughput Analysis of IEEE 802.11 Multi-hop MANETs Networks. IEEE/ACM Transaction on Networking, June,2007.

6. Safa,H.;Mirza,O.“A load balancing energy efficient clustering algorithm for MANETs”, International Journal Of Communication Systems, Vol.23, Issue4, pp .463-483, April2010.

7. Hong mei Deng,Wei Li, and Dharma P.Agrawal, “ Routing Security in Wireless Ad Hoc Network, " IEEE Communications Magazine, vol.40,no.10,October2002.

8. Dekar,L.,\&Kheddouci,H.,(2008).AClusterBasedMobilityPredictionSchemeforAdHocNetworks",AdHocNetworksJour nal.Vol.6(2),April2008,Elsevier.

9. Dimitrios Liarokapis, and Ali Shahrabi "An Adaptive Broadcasting Scheme in Mobile Ad Hoc Networks" 2009 IEEE Communication Networks and Services Research Conference, 2009. CNSR '09. Seventh Annual.

10. S.Jiang,"An enhanced prediction-based link availability estimation for MANETs, "IEEE Transactions on Communications, vol.52,no.2,pp.183-186,2004.

11. Rodoplu,V.\& Teresa,H. (1999) .Minimum Energy Mobile Wireless Networks. IEEE Journal on selected areas in communications, 17(8),1333-1344.

12. Feng,Wei;Elmirghani,JaafarM.H."Life time evaluation in energy-efficient rectangular ad hoc wireless networks", International Journal of Communication Systems, Vol.23, Issue12, pp.1632-1650, Dec2010.

13. Xia oxia Huang, Yuguang Fang. Performance Study of Node-Disjoint Multi-path Routing in Vehicular MANETs Networks. IEEE Transactions on Vehicular Technology, vol.58, pages1942-1950,May2009.

14. C.Siva Rama Murthy, B.S.Manoj, "Ad Hoc Wireless Networks: Architectures and Protocols", Prentice Hall publishers,May2004,ISBN013147023X

15. B. Johnson and D.A.Maltz, " Dynamic source routing in ad hoc wireless networks, " in Mobile Computing, T. Imielinski and H.Korth,Eds.Boston, MA: Kluwer Academic,1996,ch.5,pp.153-181.

16. S.M.Jiang,Y.D.Liu,Y.M.Jiang, and Q.H.Yin, "Provisioning of adaptability to variable topologies for routing schemes in MANETs, " IEEE J.Sel. Areas Commun.,vol.22,no.7,pp.1347-1356,Sep.2004.

17. F.F.Zou,X.M.Zhang,X.M.Gao,D.Shi,E.B.Wang,"Load balance routing using packet success rate fo rmobile ad hoc networks", Proc. Of the $3^{\text {rd }}$ IEEE International Conference on Wireless Communications, Networking and Mobile Computing(WICOM'07),September.2007. 
18. MengLi,LinZhang1,VictorO.K.Li,XiumingShan,YongRen,“An Energy-Aware Multipath Routing Protocol for Mobile Ad Hoc Networks” ACM Sigcomm Asia’05, Apr.10-12,2005,Beijing,China.

19. J.Hakoda,H.Uehara,andM.Yokoyama," Performance Evaluation of Mobile Ad Hoc Routing Protocols Based on Link Expiration Time and Load of Node", IEICE Trans.Commun.,vol.J85B,no.12,pp.2108-2118,Dec.2002.

20. Wu,Chien-Min;Hou,Ting-Chao;Leou,Maw-Lin;Liaw,Yi-Ching;Chan,Ming-Chieh“'Adaptive back off scheme for ad hoc networks based on IEEE 802.11", International Journal of Communication Systems ,Vol.23,Issue12,pp.15001520,Dec2010 